\title{
Small Signal Dynamic DQ Model of Modular Multilevel Converter for System Studies
}

\begin{tabular}{|r|l|}
\hline Journal: & IEEE Transactions on Power Delivery \\
\hline Manuscript ID: & TPWRD-00046-2015.R1 \\
\hline Manuscript Type: & Transactions \\
\hline Date Submitted by the Author: & $25-$ May-2015 \\
\hline Complete List of Authors: & $\begin{array}{l}\text { Jamshidifar, Ali; University of Aberdeen, School of enginnering } \\
\text { Jovcic, Dragan; University of Aberdeen, Engineering }\end{array}$ \\
\hline Technical Topic Area : & HVDC transmission and distribution < Transmission and Distribution \\
\hline Key Words: & $\begin{array}{l}\text { Power system dynamic stability, Power system modeling, Converters, } \\
\text { HVDC transmission, Power system control }\end{array}$ \\
\hline
\end{tabular}

\section{SCHOLARONE ${ }^{\text {m }}$ \\ Manuscripts}




\title{
Small Signal Dynamic DQ Model of Modular Multilevel Converter for System Studies
}

\author{
Aliakbar Jamshidi Far, Dragan Jovcic, Senior Member, IEEE
}

\begin{abstract}
This article presents three dynamic linear state space models of Modular Multilevel Converter (MMC) which are suitable for small-signal dynamic studies and controller design. The three models differ by the number of states (two, six and ten) and therefore are suitable for different applications based on the required accuracy. The $2^{\text {nd }}$ and $6^{\text {th }}$ order models ignore dynamics of the second harmonics and circulating current suppression control. The main challenges of dynamic analytical modelling of MMC are the non-linear multiplication terms in equations for modulating oscillating signals. The multiplication non-linear terms is therefore considered directly in the rotating DQ frame. This requires simultaneous modeling in zero sequence, fundamental frequency $D Q$ and double fundamental frequency DQ2 frames. The proposed linear analytical models are implemented in state-space in MATLAB. The validity and accuracy of the models are verified against detailed 401 level MMC model in PSCAD/EMTDC in both: time and frequency domains. The results show very good accuracy for the $10^{\text {th }}$ order model and decreasing accuracy for the lower order models.
\end{abstract}

Index Terms-Power system dynamics, Power System Modeling, Converters, HVDC transmission, Power System Control.

\section{INTRODUCTION}

Modular Multilevel Converter (MMC) has become one of the preferred topologies for VSC based High Voltage Direct Current (HVDC) applications [1]-[3].

The detailed non-linear dynamic models of MMC with different modulation techniques are presented in [2], [4]. These models are capable of representing cell-level dynamics and events. However they are discrete in nature and require a considerable amount of simulation time. The model complexity and computation burden will increase when the number of levels increases. In particular when transmission systems with multiple converters are studied, like DC grids, detailed celllevel models are not convenient.

The average MMC models [5], [6] are introduced to improve the simulation speed of MMC models. The aim of average modeling is to replicate the average response between switching instants by using mathematical equations and controlled voltage or current sources. These MMC average models are represented in static $\mathrm{ABC}$ frame which are not suitable for analytical studies. The ABC frame models use oscillating variables and support only trial and error studies with simulation in time domain. On the other hand, transferring the MMC ABC average model to DQ frame cannot be done directly because of complexity of multiplication terms caused by modulating signals and harmonic coupling in the dynamic equations.

This project is funded by European Research Council under the Ideas program in FP7; grant no 259328, 2010.

The authors are with the School of Engineering, University of Aberdeen, Aberdeen, AB24 3UE, U.K. (ajamshidifar@abdn.ac.uk, d.jovcic@abdn.ac.uk,).
The dynamic phasors can be employed for MMC average modeling as in [7], but they will result in very high number of dynamic equations.

A convenient Phasor MMC model has been developed recently [8], but Phasor modeling neglects all the dynamics.

This paper aims developing an analytical dynamic MMC model that is convenient for small signal MMC stability studies, and control design. The complex HVDC systems have numerous control loops and require accurate small-signal models for multivariable analytical control design [9], [10]. DQ frame modeling will be chosen to enable state-space model form which can support eigenvalue studies. The DQ modeling also has advantage over $\mathrm{ABC}$ average modeling in terms of simulation speed as studied in [11]. However, the model will have limitations as with all small-signal models, and it cannot be used with control non-linearities or large signal inputs.

The MMC state space model will be interlinked with other subsystems such as AC, PLL and control subsystems. A modular modeling approach is adopted to reduce complexity and enable study of subsystem interactions.

\section{MMC NONLIEAR MODEL IN ABC FRAME}

Fig.1 shows the structure of one phase leg of MMC [1],[5], [6]. It consists of two arms (positive and negative) per each phase $(x)$. Each arm includes $N$ sub-modules (SMs), one equivalent resistor $R_{\text {armx }}$, and one inductor $L_{a r m x}$ which is required to filter arm currents independently.

An average dynamic model for MMC is developed by substituting the arm SMs with an equivalent controlled voltage source as shown in Fig.1:

$\frac{d}{d t}\left[\begin{array}{c}i_{\text {diff }} \\ v_{\mathrm{CP}}^{\Sigma} \\ v_{\mathrm{CN}}^{\Sigma}\end{array}\right]=\left[\begin{array}{ccc}-\frac{R_{\text {arm }}}{L_{\text {arm }}} & \frac{m_{\mathrm{P}}}{2 L_{\text {arm }}} & \frac{m_{\mathrm{N}}}{2 L_{\text {arm }}} \\ \frac{-m_{\mathrm{P}}}{C_{a r m}} & 0 & 0 \\ \frac{-m_{\mathrm{N}}}{C_{a r m}} & 0 & 0\end{array}\right]\left[\begin{array}{c}i_{\text {diff }} \\ v_{\mathrm{CP}}^{\Sigma} \\ v_{\mathrm{CN}}^{\Sigma}\end{array}\right]+\left[\begin{array}{c}\frac{-V_{\mathrm{DC}}}{2 L_{\text {arm }}} \\ \frac{-m_{\mathrm{P}} i_{\mathrm{V}}}{2 C_{a r m}} \\ \frac{m_{\mathrm{N}} i_{\mathrm{V}}}{2 C_{a r m}}\end{array}\right]$

where $C_{a r m}=C / N, C$ is the capacitance of one $\mathrm{SM}, \mathrm{i}_{\text {diff }}$ is the differential current, $v_{C P}$ and $v_{C N}$ are the positive and negative pole voltages, $v_{\mathrm{CP}}^{\Sigma}$ and $v_{\mathrm{CN}}^{\Sigma}$ are equivalent sum (maximal) voltages of positive and negative arms, $i_{v}$ is the converter ac side current, $V_{D C}$ and $I_{D C}$ are the DC bus voltage and current, and $m_{P}$ and $m_{N}$ are modulation indices of corresponding arms. Fig. 2 shows a structural diagram of MMC model connected to $\mathrm{AC}$ and $\mathrm{DC}$ systems, where the converter $\mathrm{AC}$ voltage $e_{x}$ and DC current $I_{D C}$ are given by [5],[6]: 


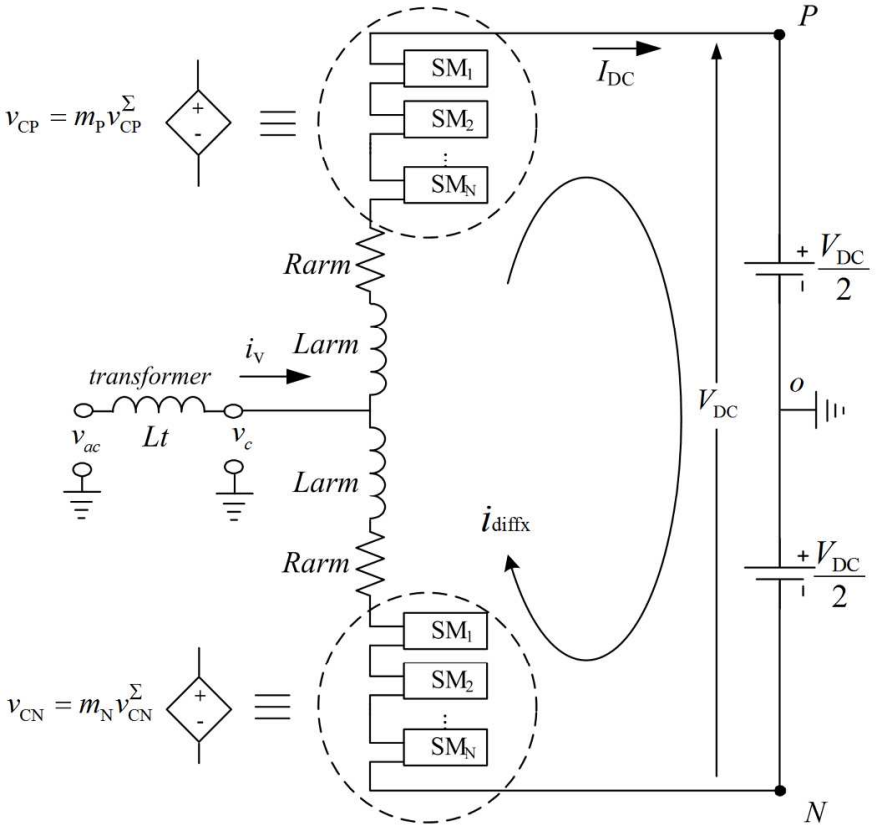

Fig.1. Circuit diagram of one phase (x) leg of MMC

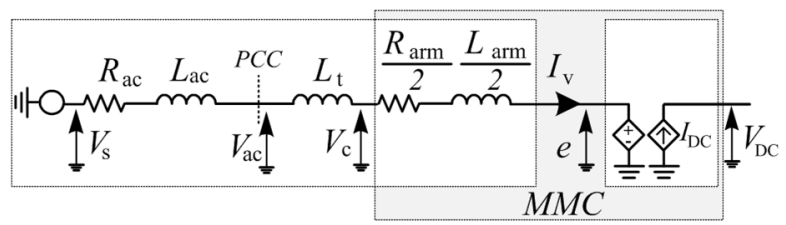

Fig. 2. Structural diagram of the average MMC model.

$$
\begin{aligned}
& e_{x}=\frac{1}{2}\left(m_{N x} v_{C N x}^{\Sigma}-m_{P x} v_{C P x}^{\Sigma}\right) \\
& I_{\mathrm{DC}}=\sum_{x=a, b, c} i_{\text {diffx }}=i_{\text {diff a }}+i_{\text {diff } \mathrm{b}}+i_{\text {diff } \mathrm{c}}
\end{aligned}
$$

\section{MMC NONLIEAR MODEL IN DQ FRAME}

The inputs to the MMC model in rotating DQ frame are assumed to be: $d q$ components of the AC current $i_{v}$, DC voltage $V_{D C}$ and $d q$ components of the control signal $m$. The outputs of the model are DC current $I_{D C}$ and $d q$ components of the converter AC voltage $e$.

\section{A. Assumptions}

The standard MMC average modeling assumptions are:

- Control modulation index $m(t)$, is a fundamental sine signal $\left(M_{d}, M_{q}\right)$ with additional second harmonic $\left(M_{d 2}\right.$, $M_{q 2}$ ) which is studied in section IV.A,

- AC current $i_{v}(t)$, is a fundamental sine signal,

- Differential current $i_{\text {diff }}(t)$, is DC plus second harmonic,

- Sum capacitor voltages $v_{C P}^{\Sigma}(t), v_{C N}^{\Sigma}(t)$, are DC, fundamental component and second harmonic.

These signals are represented as follows:

$$
\begin{aligned}
& m_{P}=\frac{1}{2}\left(1-M \cos \left(\omega t-\theta_{m}\right)-M_{2} \cos \left(2 \omega t-\theta_{m 2}\right)\right)=\left(\frac{1}{2}\right)_{0}+\left(-\frac{M_{d}}{2}\right)_{\mathrm{d}} \cos (\omega t) \\
& +\left(-\frac{M_{q}}{2}\right)_{\mathrm{q}} \sin (\omega t)+\left(-\frac{M_{d 2}}{2}\right)_{\mathrm{d} 2} \cos (2 \omega t)+\left(-\frac{M_{q 2}}{2}\right)_{\mathrm{q} 2} \sin (2 \omega t) \\
& m_{N}=\frac{1}{2}\left(1+M \cos \left(\omega t-\theta_{m}\right)-M_{2} \cos \left(2 \omega t-\theta_{m 2}\right)\right)=\left(\frac{1}{2}\right)_{0}+\left(\frac{M_{d}}{2}\right)_{\mathrm{d}} \cos (\omega t) \\
& +\left(\frac{M_{q}}{2}\right)_{\mathrm{q}} \sin (\omega t)+\left(-\frac{M_{d 2}}{2}\right)_{\mathrm{d} 2} \cos (2 \omega t)+\left(-\frac{M_{q 2}}{2}\right)_{\mathrm{q} 2} \sin (2 \omega t) \\
& i_{V}(t)=I_{V m} \cos \left(\omega t-\theta_{I}\right)=\left(i_{V}\right)_{d}+\left(i_{V}\right)_{q} \\
& i_{\text {diff }}(t)=I_{\text {diff } 0}+i_{\text {diff } 2} \cos \left(2 \omega t-\theta_{\text {Idiff } 2}\right)=I_{\text {diff } 0}+\left(i_{\text {diff }}\right)_{d 2}+\left(i_{\text {diff }}\right)_{q 2} \\
& v_{C P}^{\sum}(t)=\left(V_{C P}^{\sum}\right)_{0}+V_{C P m}^{\sum} \cos \left(\omega t+\theta_{V P}\right)+V_{C P m 2}^{\sum} \cos \left(2 \omega t+\theta_{V P 2}\right) \\
& =V_{C P 0}^{\sum}+\left(v_{C P}^{\sum}\right)_{d}+\left(v_{C P}^{\sum}\right)_{q}+\left(v_{C P}^{\Sigma}\right)_{d 2}+\left(v_{C P}^{\sum}\right)_{q 2} \\
& v_{C N}^{\sum}(t)=\left(V_{C N}^{\sum}\right)_{0}+V_{C N m}^{\sum} \cos \left(\omega t+\theta_{V N}\right)+V_{C N m}^{\sum} \cos \left(2 \omega t+\theta_{V N 2}\right) \\
& =V_{C N 0}^{\sum}+\left(v_{C N}^{\sum}\right)_{d}+\left(v_{C N}^{\sum}\right)_{q}+\left(v_{C N}^{\Sigma}\right)_{d 2}+\left(v_{C N}^{\Sigma}\right)_{q 2}
\end{aligned}
$$

where subscripts $d, q$ denote the two components in the coordinate frame rotating at fundamental frequency $\omega_{0}=2 \pi f$, while subscripts $d 2, q 2$ denote the two components in the coordinate frame rotating at second harmonic $2 \omega_{0}$, and subscript 0 denotes zero sequence component.

\section{B. Basic frequency and zero sequence dynamics in $D Q$ frame}

The sum capacitor voltages and differential current of (1) can be represented as:

$$
\begin{aligned}
& \frac{d}{d t}\left[\begin{array}{l}
v_{\mathrm{CPx}}^{\Sigma} \\
v_{\mathrm{CNx}}^{\Sigma}
\end{array}\right]=\left[\begin{array}{ll}
0 & 0 \\
0 & 0
\end{array}\right]\left[\begin{array}{l}
v_{\mathrm{CPx}}^{\Sigma} \\
v_{\mathrm{CNx}}^{\Sigma}
\end{array}\right]+\frac{1}{C_{a r m}}\left[\begin{array}{l}
-m_{\mathrm{Px}} i_{\mathrm{diffx}}-\frac{m_{\mathrm{Px}} i_{\mathrm{Vx}}}{2} \\
-m_{\mathrm{Nx}} i_{\mathrm{diffx}}+\frac{m_{\mathrm{Nx}} i_{\mathrm{Vx}}}{2}
\end{array}\right] \\
& \frac{d}{d t}\left[i_{\text {diffx }}\right]=\left[-\frac{R_{a r m}}{L_{a r m}}\right] i_{\text {diffx }}+\frac{1}{4 L_{a r m}}\left[-2 V_{\mathrm{DC}}+m_{\mathrm{Px}} v_{\mathrm{CPx}}^{\Sigma}+m_{\mathrm{Nx}} v_{\mathrm{CNx}}^{\Sigma}\right]
\end{aligned}
$$

Transferring equation (8) to DQ0 frame results:

$$
\frac{d}{d t}\left[\begin{array}{c}
V_{\mathrm{CPd}}^{\Sigma} \\
V_{\mathrm{CNd}}^{\Sigma} \\
V_{\mathrm{CPq}}^{\Sigma} \\
V_{\mathrm{CNq}}^{\Sigma} \\
V_{\mathrm{CP} 0}^{\Sigma} \\
V_{\mathrm{CN} 0}^{\Sigma}
\end{array}\right]=\left[\begin{array}{cccccc}
0 & 0 & \omega_{0} & 0 & 0 & 0 \\
0 & 0 & 0 & \omega_{0} & 0 & 0 \\
-\omega_{0} & 0 & 0 & 0 & 0 & 0 \\
0 & -\omega_{0} & 0 & 0 & 0 & 0 \\
0 & 0 & 0 & 0 & 0 & 0 \\
0 & 0 & 0 & 0 & 0 & 0
\end{array}\right]\left[\begin{array}{c}
V_{\mathrm{CPd}}^{\Sigma} \\
V_{\mathrm{CNd}}^{\Sigma} \\
V_{\mathrm{CPq}}^{\Sigma} \\
V_{\mathrm{CNq}}^{\Sigma} \\
V_{\mathrm{CP} 0}^{\Sigma} \\
V_{\mathrm{CN} 0}^{\Sigma}
\end{array}\right]+\mathrm{F}
$$$$
\frac{d}{d t}\left[\begin{array}{c}
I_{\text {diffd }} \\
I_{\text {diffa }} \\
I_{\text {diffo }}
\end{array}\right]=\left[\begin{array}{ccc}
-\frac{R_{a r m}}{L_{a r m}} & \omega_{0} & 0 \\
-\omega_{0} & -\frac{R_{a r m}}{L_{a r m}} & 0 \\
0 & 0 & -\frac{R_{a r m}}{L_{a r m}}
\end{array}\right]\left[\begin{array}{c}
I_{\text {diffd }} \\
I_{\text {diff }} \\
I_{\text {diffo }}
\end{array}\right]+\mathrm{k}
$$

where $\mathrm{F}, \mathrm{k}$ are the $d q 0$ components of nonlinear terms in (8). 
Assumption (5) implies that the circulating current has no basic frequency $d q$ components, therefore $I_{\text {diffd }}=I_{\text {diffq }}=0$.

The non-linear terms $\mathrm{F}, \mathrm{k}$ are products of oscillating signals and it is required to obtain their $d q$ components. This is mathematically represented for a generic signals as shown in Appendix I. By expanding every product using expression (31) in Appendix I, the matrices $\mathrm{F}$ and $\mathrm{k}$ will become:

$$
\begin{aligned}
& \mathrm{F}=\frac{1}{4 C_{\text {arm }}}\left[\begin{array}{c}
2 M_{d} I_{\mathrm{diff}}-I_{\mathrm{Vd}} \\
-2 M_{d} I_{\mathrm{diff0}}+I_{\mathrm{Vd}} \\
2 M_{q} I_{\mathrm{diff}}-I_{\mathrm{Vq}} \\
-2 M_{q} I_{\mathrm{diff} 0}+I_{\mathrm{Vq}} \\
\frac{1}{2} M_{d} I_{\mathrm{Vd}}+\frac{1}{2} M_{q} I_{\mathrm{Vq}}-2 I_{\mathrm{diff0}} \\
\frac{1}{2} M_{d} I_{\mathrm{Vd}}+\frac{1}{2} M_{q} I_{\mathrm{Vq}}-2 I_{\mathrm{diff}}
\end{array}\right] \\
& \mathrm{k}=\left[\begin{array}{c}
0 \\
0 \\
\frac{1}{4 L_{\text {arm }}}\left(-2 V_{\mathrm{DC}}+2 V_{\mathrm{CP} 0}^{\Sigma}-M_{d} V_{\mathrm{CPd}}^{\Sigma}-M_{q} V_{\mathrm{CPq}}^{\Sigma}\right)
\end{array}\right]
\end{aligned}
$$

Similarly, the output equations

(2) in DQ frame are

$\left[\begin{array}{c}E_{\mathrm{d}} \\ E_{q}\end{array}\right]=\frac{1}{4}\left[\begin{array}{l}2 M_{d} V_{\mathrm{CP} 0}^{\Sigma}-2 V_{\mathrm{CPd}}^{\Sigma}+M_{d} V_{\mathrm{CPd} 2}^{\Sigma}+M_{q} V_{\mathrm{CPq} 2}^{\Sigma} \\ 2 M_{q} V_{\mathrm{CP} 0}^{\Sigma}-2 V_{\mathrm{CPq}}^{\Sigma}-M_{q} V_{\mathrm{CPd} 2}^{\Sigma}+M_{d} V_{\mathrm{CP} 2}^{\Sigma}\end{array}\right]$
$I_{\mathrm{DC}}=3 I_{\mathrm{diff} 0}$

\section{Second harmonic dynamics for sum capacitor voltages}

Equation (11) implies that the second harmonics will influence fundamental MMC AC voltages and therefore appropriate expressions for second harmonic sum capacitor voltages are needed. Equation (8) applies also to the second harmonic, and therefore the second harmonic sum capacitor voltages $V_{\mathrm{CPd} 2}^{\Sigma}, V_{\mathrm{CPq} 2}^{\Sigma}$ are similar to (9):

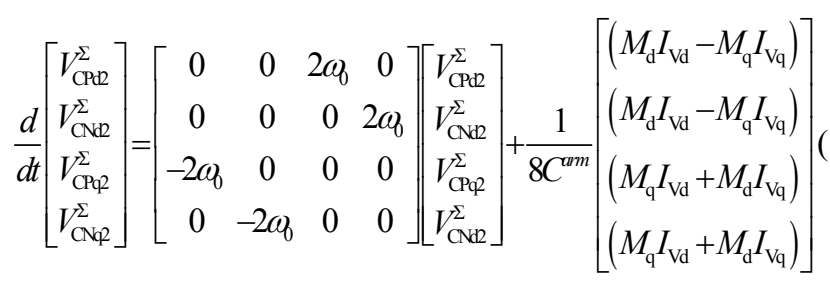

From (9), (10)and (12), it is concluded that:

$V_{\mathrm{CPd}}^{\Sigma}=-V_{\mathrm{CNd}}^{\Sigma}, V_{\mathrm{CPq}}^{\Sigma}=-V_{\mathrm{CNq}}^{\Sigma}, V_{\mathrm{CP} 0}^{\Sigma}=V_{\mathrm{CN} 0}^{\Sigma}$

$V_{C P d 2}^{\Sigma}=V_{C N d 2}^{\Sigma}, \quad V_{C P q 2}^{\Sigma}=V_{C N q 2}^{\Sigma}$

\section{D. $6^{\text {th }}$ order nonlinear dynamic MMC model}

By combining (9), (11) and (12), the nonlinear dynamic $6^{\text {th }}$ order model of MMC in DQ frame is:

$$
\begin{gathered}
\frac{d}{d t} x_{6}=\mathrm{A}_{6} x_{6}+\left[\begin{array}{c}
\left(M_{d} I_{\mathrm{Vd}}-M_{q} I_{\mathrm{Vq}}\right) / 8 C_{a r m} \\
\left(M_{q} I_{\mathrm{Vd}}+M_{d} I_{\mathrm{Vq}}\right) / 8 C_{a r m} \\
\left(2 M_{d} I_{\mathrm{diff} 0}-I_{\mathrm{Vd}}\right) / 4 C_{a r m} \\
\left(2 M_{q} I_{\mathrm{diff} 0}-I_{\mathrm{Vq}}\right) / 4 C_{a r m} \\
\left(M_{d} I_{\mathrm{Vd}}+M_{q} I_{\mathrm{Vq}}-4 I_{\mathrm{diff} 0}\right) / 8 C_{a r m} \\
\left(-2 V_{\mathrm{DC}}-M_{d} V_{\mathrm{CPd}}^{\Sigma}-M_{q} V_{\mathrm{CPq}}^{\Sigma}+2 V_{\mathrm{CP} 0}^{\Sigma}\right) / 4 L_{a r m}
\end{array}\right] \\
y=\left[\begin{array}{c}
E_{d} \\
E_{q} \\
I_{\mathrm{DC}}
\end{array}\right]=\left[\begin{array}{c}
\left(2 M_{d} V_{\mathrm{CP} 0}^{\Sigma}+M_{d} V_{\mathrm{CP} 2}^{\Sigma}+M_{q} V_{\mathrm{CPq} 2}^{\Sigma}-2 V_{\mathrm{CPd}}^{\Sigma}\right) / 4 \\
\left(2 M_{q} V_{\mathrm{CP} 0}^{\Sigma}-M_{q} V_{\mathrm{CPd} 2}^{\Sigma}+M_{d} V_{\mathrm{CPq} 2}^{\Sigma}-2 V_{\mathrm{CPq}}^{\Sigma}\right) / 4 \\
3 I_{\mathrm{diff} 0}
\end{array}\right]
\end{gathered}
$$

where:

$$
\begin{aligned}
x_{6} & =\left[\begin{array}{cccccc}
V_{\mathrm{CPd} 2}^{\Sigma} & V_{\mathrm{CP} 2}^{\Sigma} & V_{\mathrm{CPd}}^{\Sigma} & V_{\mathrm{CPq}}^{\Sigma} & V_{\mathrm{CP} 0}^{\Sigma} & I_{\mathrm{diff} 0}
\end{array}\right]^{T} \\
\mathrm{~A}_{6} & =\left[\begin{array}{cccccc}
0 & 2 \omega_{0} & 0 & 0 & 0 & 0 \\
-2 \omega_{0} & 0 & 0 & 0 & 0 & 0 \\
0 & 0 & 0 & \omega_{0} & 0 & 0 \\
0 & 0 & -\omega_{0} & 0 & 0 & 0 \\
0 & 0 & 0 & 0 & 0 & 0 \\
0 & 0 & 0 & 0 & 0 & -\frac{R_{\text {arm }}}{L_{\text {arm }}}
\end{array}\right]
\end{aligned}
$$

This model includes second harmonic voltages but does not include second harmonic current or harmonic suppression control dynamics. It can be used under the assumption that circulating current suppression control totally eliminates current harmonics without any side-impact on power flow. As it will be shown, this model can be used for MMC dynamic modeling but with limited accuracy.

\section{NONLINEAR MODEL WITH CCSC}

\section{A. Circuating current second harmonic model}

The dynamics of second harmonic circulating current can be derived using (8) in DQ2 frame rotating at $2 \omega_{0}$ as:

$$
\begin{aligned}
& \frac{d}{d t}\left[\begin{array}{l}
I_{\text {diff } 2} \\
I_{\text {diff } 2}
\end{array}\right]=\left[\begin{array}{cc}
0 & 2 \omega_{0} \\
-2 \omega_{0} & 0
\end{array}\right]\left[\begin{array}{c}
I_{\text {diffd } 2} \\
I_{\text {diffq } 2}
\end{array}\right]-\frac{R_{\text {arm }}}{L_{\text {arm }}}\left[\begin{array}{c}
I_{\text {diffd } 2} \\
I_{\text {diff } 22}
\end{array}\right] \\
& +\frac{1}{2 L_{\text {arm }}}\left[\begin{array}{l}
\left(-V_{\mathrm{DC}}+m_{\mathrm{Px}} V_{\mathrm{CPx}}^{\Sigma}+m_{\mathrm{Nx}} V_{\mathrm{CNx}}^{\Sigma}\right)_{d 2} \\
\left(-V_{\mathrm{DC}}+m_{\mathrm{Px}} V_{\mathrm{CPx}}^{\Sigma}+m_{\mathrm{Nx}} V_{\mathrm{CNx}}^{\Sigma}\right)_{q 2}
\end{array}\right]
\end{aligned}
$$

This expression is expanded considering (31) as:

$$
\begin{aligned}
& \frac{d}{d t}\left[\begin{array}{l}
I_{\text {diffd } 2} \\
I_{\text {diff } 22}
\end{array}\right]=\left[\begin{array}{cc}
0 & 2 \omega_{0} \\
-2 \omega_{0} & 0
\end{array}\right]\left[\begin{array}{l}
I_{\text {diffd } 2} \\
I_{\text {diff } 2}
\end{array}\right]-\frac{R_{\text {arm }}}{L_{\text {arm }}}\left[\begin{array}{c}
I_{\text {diffd } 2} \\
I_{\text {diff } 2}
\end{array}\right] \\
& +\frac{1}{4 L_{\text {arm }}}\left[\begin{array}{l}
-M_{d} V_{\mathrm{CPd}}^{\Sigma}+M_{q} V_{\mathrm{CPq}}^{\Sigma}+2 V_{\mathrm{CPd} 2}^{\Sigma}-2 M_{d 2} V_{\mathrm{CP} 0}^{\Sigma} \\
-M_{q} V_{\mathrm{CPd}}^{\Sigma}-M_{d} V_{\mathrm{CPq}}^{\Sigma}+2 V_{\mathrm{CPq} 2}^{\Sigma}-2 M_{q 2} V_{\mathrm{CP} 0}^{\Sigma}
\end{array}\right]
\end{aligned}
$$




\section{B. CCSC (Circulating Current Suppression Control) model}

The control signals $M_{d 2}$ and $M_{q 2}$ are the outputs of PI control loops to suppress $I_{\text {diffdz }}$ and $I_{\text {diffq } 2}$ as shown in Fig. 3 [12]. From Fig. 3, the dynamic equations of $M_{d 2}$ and $M_{q 2}$ are:

$$
\frac{d}{d t}\left[\begin{array}{l}
M_{d 2} \\
M_{q 2}
\end{array}\right]=-K_{I}\left[\begin{array}{l}
I_{d i f d z 2} \\
I_{d i f f a 2}
\end{array}\right]-K_{P} \frac{d}{d t}\left[\begin{array}{l}
I_{d i f f d 2} \\
I_{d i f f i 2}
\end{array}\right]
$$

By substituting (17) in (18):

$$
\begin{aligned}
& \frac{d}{d t}\left[\begin{array}{l}
M_{d 2} \\
M_{q 2}
\end{array}\right]=\left[\begin{array}{cc}
K_{I}-K_{P} R_{a m} / L_{a m m} & 2 K_{P} \omega_{q} \\
-2 K_{P} \omega_{q} & K_{I}-K_{P} R_{a m} / L_{a m m}
\end{array}\right]\left[\begin{array}{c}
I_{d i f f d 2} \\
I_{d i f f q 2}
\end{array}\right] \\
& +\frac{K_{P}}{4 L_{a m m}}\left[\begin{array}{l}
-M_{d} V_{\mathrm{CPd}}^{\Sigma}+M_{q} V_{\mathrm{CPq}}^{\Sigma}+2 V_{\mathrm{CP} 12}^{\Sigma}-2 M_{d 2} V_{\mathrm{CP}}^{\Sigma} \\
-M_{q} V_{\mathrm{CPd}}^{\Sigma}-M_{d} V_{\mathrm{CPq}}^{\Sigma}+2 V_{\mathrm{CPR} 2}^{\Sigma}-2 M_{q 2} V_{\mathrm{PP} 0}^{\Sigma}
\end{array}\right]
\end{aligned}
$$

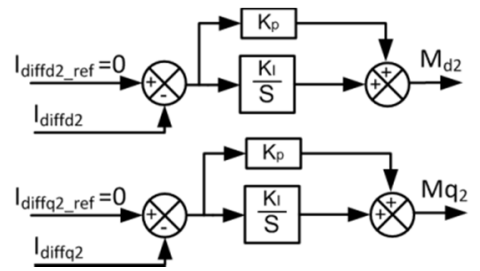

Fig. 3. CCSC (PI control loops for suppressing $I_{\text {diffd } 2}$ and $I_{d i f f q_{2}}$ )

\section{C. $10^{\text {th }}$ order nonlinear dynamic model}

The proposed $10^{\text {th }}$ order MMC model consists of the $6^{\text {th }}$ order model (14) and the four states of (17), (19). However, the $6^{\text {th }}$ order model needs to be revised by considering the second harmonic terms of circulating current and modulation indices. The $6^{\text {th }}$ order equations(14) and (15) are revised as:

$$
\begin{aligned}
& \frac{d}{d t} x_{6}=\mathrm{A}_{6} x_{6}+\mathbf{Z}_{6} \\
& y_{10}=\left[\begin{array}{c}
E_{d} \\
E_{q} \\
I_{\mathrm{DC}}
\end{array}\right]= \\
& {\left[\begin{array}{c}
\left(2 M_{d} V_{\mathrm{CP} 0}^{\Sigma}+M_{d} V_{\mathrm{CPd} 2}^{\Sigma}+M_{q} V_{\mathrm{CP} 2}^{\Sigma}-2 V_{\mathrm{CPd}}^{\Sigma}+M_{d 2} V_{\mathrm{CPd}}^{\Sigma}+M_{q 2} V_{\mathrm{CPq}}^{\Sigma}\right) / 4 \\
\left(2 M_{q} V_{\mathrm{CP} 0}^{\Sigma}-M_{q} V_{\mathrm{CP} 2}^{\Sigma}+M_{d} V_{\mathrm{CP} 2}^{\Sigma}-2 V_{\mathrm{CPq}}^{\Sigma}-M_{d 2} V_{\mathrm{CPq}}^{\Sigma}+M_{q 2} V_{\mathrm{CPd}}^{\Sigma}\right) / 4 \\
3 I_{\mathrm{diff} 0}
\end{array}\right]}
\end{aligned}
$$

Where the input terms are:

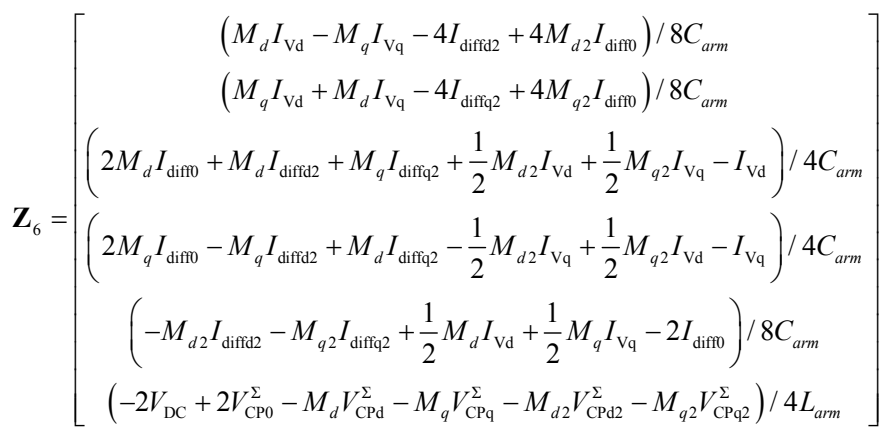

By combining (17),(19) and (20), the $10^{\text {th }}$ order nonlinear model in DQ frame is derived:

$$
\frac{d}{d t} x_{10}=\left[\begin{array}{c|c}
\mathrm{A}_{6} & 0_{6 \times 4} \\
\hline 0_{4 \times 6} & \mathrm{~A}_{r e s}
\end{array}\right] x_{10}+\left[\frac{\mathbf{Z}_{6}}{\mathbf{Z}_{r e s}}\right], y=y_{10}
$$

where

$$
\begin{aligned}
& x_{10}=\left[\begin{array}{c}
\frac{x_{6}}{I_{\text {diffa }}} \\
I_{d i f f q_{2}} \\
M_{d 2} \\
M_{q 2}
\end{array}\right]_{10 \times 1}, \mathrm{~A}_{r e s}=\left[\begin{array}{cccc}
-\frac{R_{a r m}}{L_{\text {arm }}} & 2 \omega_{0} & 0 & 0 \\
-2 \omega_{0} & -\frac{R_{a r m}}{L_{\text {arm }}} & 0 & 0 \\
K_{I}-K_{P} \frac{R_{\text {arm }}}{L_{\text {arm }}} & 2 K_{P} \omega_{0} & 0 & 0 \\
-2 K_{P} \omega_{0} & K_{I}-K_{P} \frac{R_{\text {arm }}}{L_{\text {arm }}} & 0 & 0
\end{array}\right] \\
& \mathbf{Z}_{r e s}=\left[\begin{array}{c}
\left(-M_{d} V_{C P d}^{\Sigma}+M_{q} V_{C P q}^{\Sigma}+2 V_{C P d 2}^{\Sigma}-2 M_{d 2} V_{C P 0}^{\Sigma}\right) / 4 L_{a r m} \\
\left(-M_{d} V_{C P q}^{\Sigma}-M_{q} V_{C P q}^{\Sigma}+2 V_{C P q 2}^{\Sigma}-2 M_{q 2} V_{\mathrm{CP} 0}^{\Sigma}\right) / 4 L_{a r m} \\
K_{P}\left(-M_{d} V_{C P d}^{\Sigma}+M_{q} V_{C P q}^{\Sigma}+2 V_{C P d 2}^{\Sigma}-2 M_{d 2} V_{C P 0}^{\Sigma}\right) / 4 L_{a r m} \\
K_{P}\left(-M_{d} V_{C P q}^{\Sigma}-M_{q} V_{C P q}^{\Sigma}+2 V_{C P q 2}^{\Sigma}-2 M_{q 2} V_{C \mathrm{CP}}^{\Sigma}\right) / 4 L_{a r m}
\end{array}\right]
\end{aligned}
$$

Fig. 4 illustrates the nonlinear dynamic $10^{\text {th }}$ order MMC model (22) and (23) in the block diagram format. The static equations related to the products of $d q$ terms can be expanded using the formulae in the appendix. The model's inputs, control inputs, and outputs are also shown in the figure.

Comparing with $6^{\text {th }}$ order model, this $10^{\text {th }}$ order model includes impact of $M_{d 2}$ and $M_{q 2}$ on the fundamental equations in (20) and (21). In order to represent the magnitude of control effort $M_{d 2}$ and $M_{q 2}$, the four dynamic equations (17) and (19) must be included despite the fact that second harmonic differential currents are zero in steady-state.

\section{V. $2^{\mathrm{ND}}$ ORDER DYNAMIC MODEL WITH SERIES CAPACITANCE}

\section{A. Steady-state analysis of $M M C$}

The steady-state static model of MMC (phasor model) can be derived from either the equations (14) or (22) by equating the dynamic terms to zero. In steady-state, $I_{d i f f d 2}$ and $I_{d i f f q_{2}}$ are zero (because of CCSC) and $M_{d 2}$ and $M_{q 2}$ are very small values. Therefore, the steady-state equations from either the $6^{\text {th }}$ order or $10^{\text {th }}$ order dynamic equations give similar results.

Starting from (14) and equating the dynamic terms to zero:

$A_{s s} x_{6}=B_{s s} u, \quad y=C_{s s} x_{6}$

Where $y$ and $x_{6}$ are given in (14) and (15), and the new matrices are: 


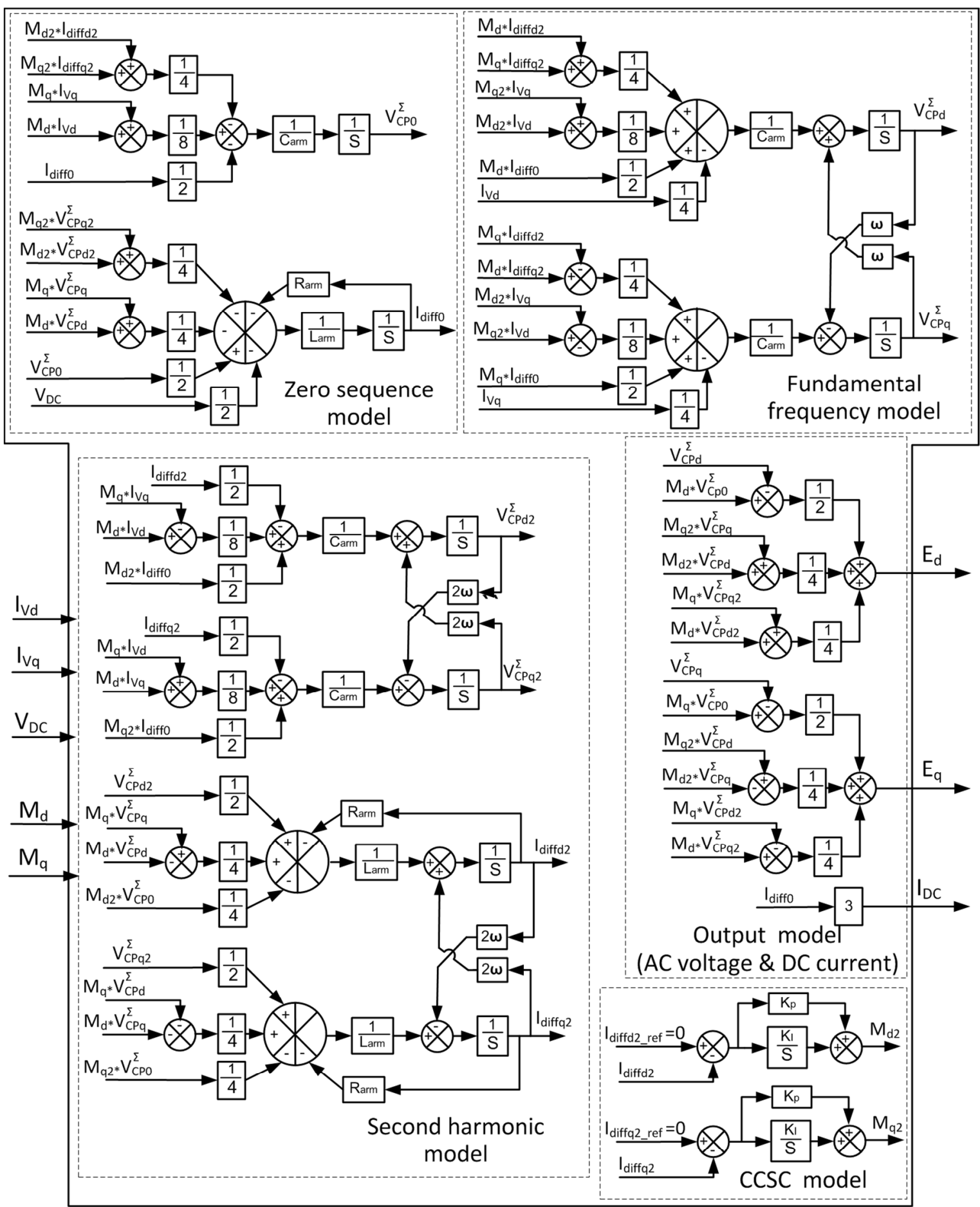

Fig. 4. MMC $10^{\text {th }}$ order dynamic DQ model

$$
A_{s s}=\left[\begin{array}{cccccc}
1 & 0 & 0 & 0 & 0 & 0 \\
0 & 1 & 0 & 0 & 0 & 0 \\
0 & 0 & 1 & 0 & 0 & -M_{q} / 2 \omega C_{a r m} \\
0 & 0 & 0 & 1 & 0 & M_{d} / 2 \omega C_{a r m} \\
0 & 0 & 0 & 0 & 1 & -2 R_{a r m} \\
0 & 0 & 0 & 0 & 0 & 1
\end{array}\right]
$$

$$
\begin{aligned}
& u=\left[\begin{array}{c}
I_{v d} \\
I_{v q} \\
V_{D C}
\end{array}\right], \\
& B_{s s}{ }^{T}=\frac{1}{16 \omega C_{a r m}}\left[\begin{array}{cccccc}
-M_{q} & M_{d} & 0 & -4 & -2 M_{q} & 4 M_{d} \omega C_{a r m} \\
M_{d} & -M_{q} & 4 & 0 & 2 M_{d} & 4 M_{q} \omega C_{a r m} \\
0 & 0 & 0 & 0 & 16 \omega C_{a r m} & 0
\end{array}\right] \\
& C_{s s}=\frac{1}{4}\left[\begin{array}{cccccc}
M_{d} & M_{q} & -2 & 0 & 2 M_{d} & 0 \\
-M_{q} & M_{d} & 0 & -2 & 2 M_{q} & 0 \\
0 & 0 & 0 & 0 & 0 & 12
\end{array}\right]
\end{aligned}
$$


The MMC AC voltages, $E_{d}$ and $E_{q}$, can be expressed by substituting (25) and (26) into the sum capacitor voltages (11). If the converter resistance in (25) is also neglected, i.e. $R_{\text {arm }}=0$, then the MMC AC voltage can be represented as follows:

$$
\begin{aligned}
& E_{d}=\frac{I_{V q}}{\omega C_{M M C}}+\frac{M_{d} V_{d c}}{2} \\
& E_{d}=-\frac{I_{V d}}{\omega C_{M M C}}+\frac{M_{q} V_{d c}}{2}
\end{aligned}
$$

where the fictitious capacitance $C_{M M C}$ is same as in [8]:

$$
C_{M M C}=64 C^{a r m} /\left(8-3\left(M_{d}^{2}+M_{q}^{2}\right)\right)
$$

If the MMC reactance $X_{M M C}=1 /\left(\omega C_{M M C}\right)$ is introduced then the phasor model from [8] is obtained.

\section{B. $2^{\text {nd }}$ order nonlinear dynamic model}

Considering the convenient and simple model in (27) it is possible to derive the $2^{\text {nd }}$ order dynamic model of MMC:

$$
\begin{aligned}
& \frac{d}{d t}\left[\begin{array}{l}
x_{d} \\
x_{q}
\end{array}\right]=\left[\begin{array}{cc}
0 & \omega \\
-\omega & 0
\end{array}\right]\left[\begin{array}{l}
x_{d} \\
x_{q}
\end{array}\right]+\frac{1}{C_{M M C}}\left[\begin{array}{c}
I_{V d} \\
I_{V q}
\end{array}\right] \\
& y=\left[\begin{array}{c}
e_{d} \\
e_{q} \\
I_{d c}
\end{array}\right]=\left[\begin{array}{c}
x_{d}+0.5 M_{d} V_{D C} \\
x_{q}+0.5 M_{q} V_{D C} \\
0.75 M_{d} I_{v d}+0.75 M_{q} I_{v q}
\end{array}\right]
\end{aligned}
$$

where $x_{d}$ and $x_{q}$ represent the voltage drop across the $C_{M M C}$ in DQ frame. The $2^{\text {nd }}$ order model shows that MMC responds like a 2-level VSC behind a series capacitance $C_{M M C}$ on the AC side, as shown in Fig. 5. This is much different dynamic topology from the modeling of 2-level VSC, where the converter capacitance is in parallel on the DC side.

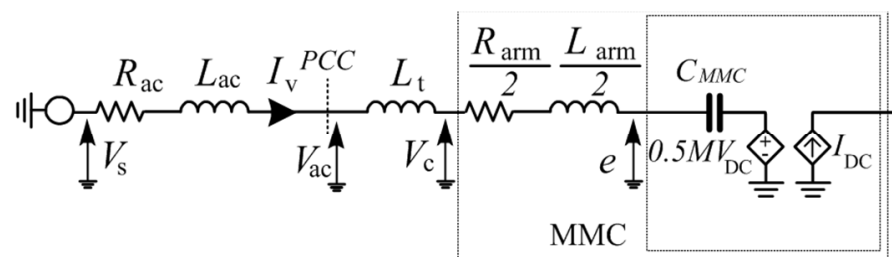

Fig. 5. Simplified $2^{\text {nd }}$ order dynamic MMC model connected to the AC grid.

\section{MODEL VERIFICATION}

\section{A. Linearized dynamic $D Q M M C$ model}

The $2^{\text {nd }}, 6^{\text {th }}$ and $10^{\text {th }}$ order nonlinear models are linearized around steady-state operating point, and interlinked with the corresponding models of AC and PLL sub-systems. The dynamic of PLL is represented by a second order state-space equation as in [13]. The final linearised models of $10^{\text {th }}, 14^{\text {th }}$ and $18^{\text {th }}$ order are implemented in MATLAB, and their accuracy is tested against detailed PSCAD Benchmark model.

\section{B. PSCAD benchmark model}

The PSCAD benchmark model consists of a MMC converter represented as given in [4], which is connected to an $\mathrm{AC}$ system and a DC source with a series resistance. The MMC is a 401-level 1000MVA converter with $C_{S M}=10 \mathrm{mF}, R_{\text {arm }}=1.2 \Omega$, and $L_{\text {arm }}=0.08 \mathrm{H}$. The $\mathrm{AC}$ grid is given by $V_{A C}=370 \mathrm{KV}$, $S C R=8.5, X / R=10, X t=8 \%$, while DC side parameters are $V_{D C}=640 \mathrm{KV}$ with series resistance of $R_{D C}=0.5 \Omega$. The CCSC controller gains are $K_{P}=0.5$ and $K_{I}=50$.

\section{Verification of the models in time domain}

The $10^{\text {th }}$ order linearized model is compared against the benchmark PSCAD model for a $5 \%$ step up on control input $M_{d}$ as shown in Fig. 6. The matching is excellent for all AC, DC and control variables, and verifies accuracy of the model.
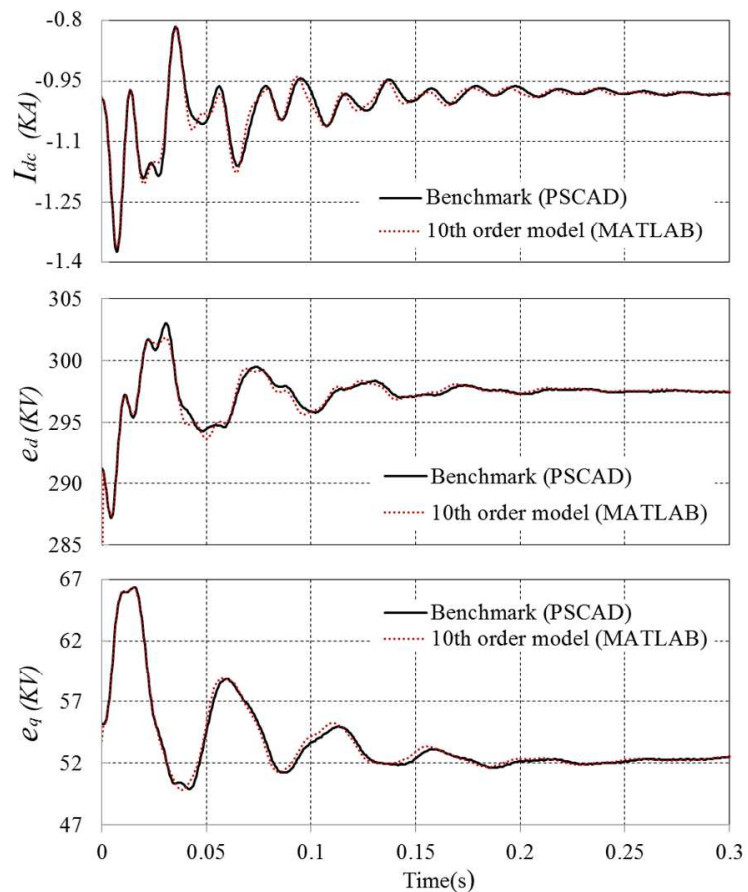

Fig. 6. Comparison of the $10^{\text {th }}$ order linearized MMC model against PSCAD benchmark model for $5 \%$ step up on $M_{d}$

The accuracy of the two lower order models is also tested for the same step input as shown in Fig. 7. As it can be seen the lower order models show less accuracy as expected, but might be able to capture dominant MMC oscillatory mode.

\section{Verification of the models in frequency domain}

For the purpose of accurate verification, the models are also tested in frequency domain. PSCAD has no frequency domain analysis capability, and the results are obtained manually, by injecting a single frequency component at a time.

Fig. 8 shows the frequency response comparison of the $10^{\text {th }}$ order and benchmark models in frequency range $1-150 \mathrm{~Hz}$. Very good matching is seen across the entire frequency range.

Fig. 9 shows the frequency response of $2^{\text {nd }}$ and $6^{\text {th }}$ order models against the benchmark model in the same frequency range. It can be seen that the $6^{\text {th }}$ order model shows acceptable matching especially in the frequency range below $20 \mathrm{~Hz}$. 

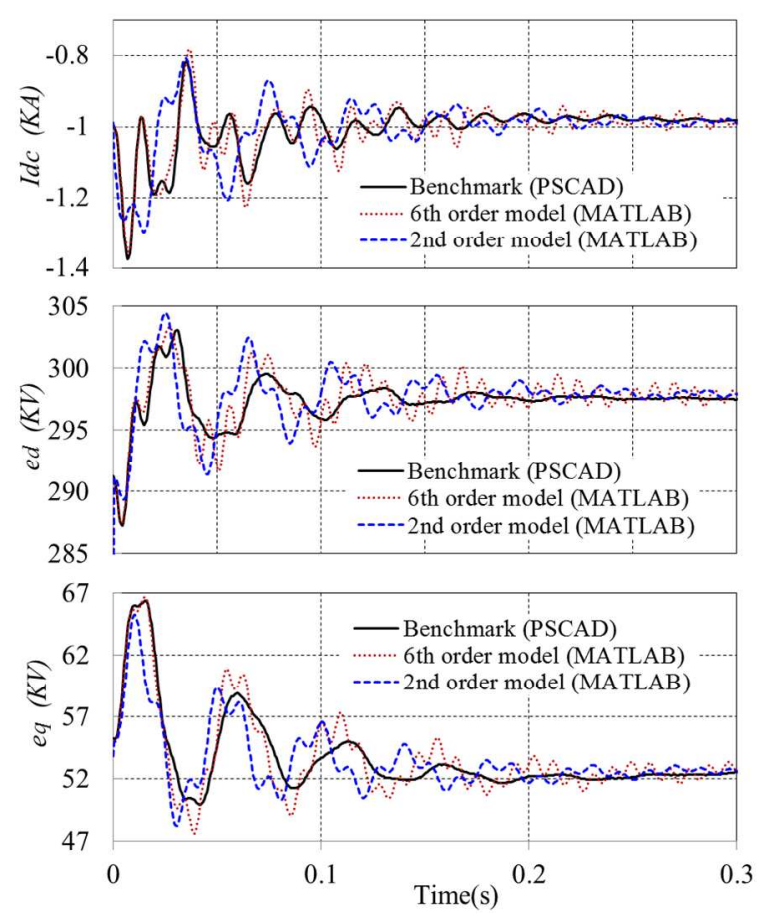

Fig. 7. Comparison of the $2^{\text {nd }}$ and $6^{\text {th }}$ order linearized MMC models against PSCAD benchmark model for $5 \%$ step up on $M_{d}$

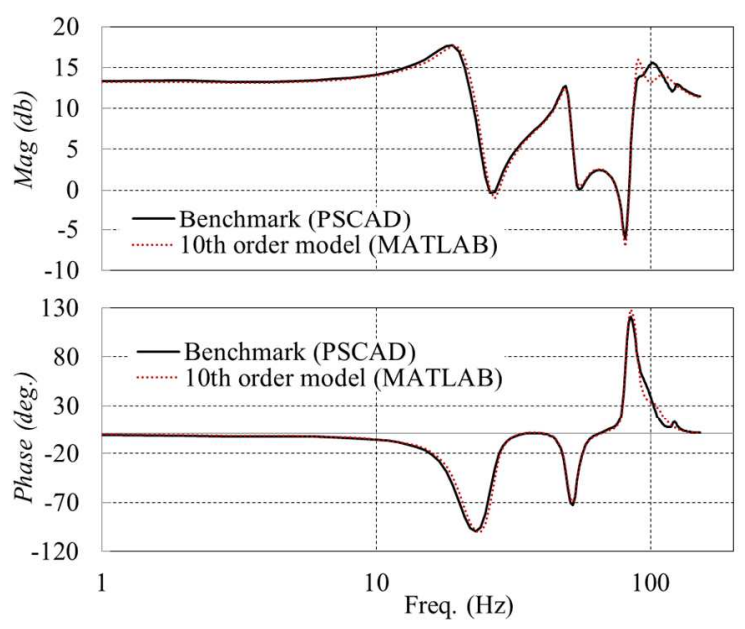

Fig. 8. Frequency response comparison for $10^{\text {th }}$ order model
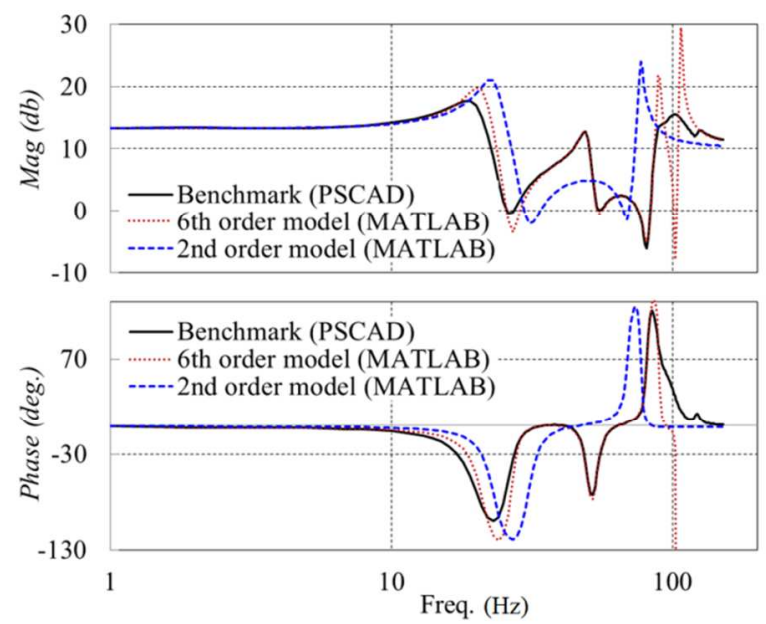

Fig. 9. Frequency response for $2^{\text {th }}$ and $6^{\text {th }}$ order models

\section{E. Dynamic study of influence of PLL gains}

The proposed models are suitable for wide range of $\mathrm{MMC}$ small-signal dynamic studies. As an example, Fig. 10 shows the effect of increasing the PLL (Phase locked loop) gains by a factor of 10, and Table I shows the set of dominant eigenvalues (out of 18 eigenvalues). High PLL gains might be desired for good post fault synchronisation, however it is seen that the system suffers from new lightly damped oscillatory mode around $50 \mathrm{~Hz}$ when PLL gains are increased.

Table I. Set of dominant eigenvalues for different PLL and CCSC gains.

\begin{tabular}{|c|c|c|}
\hline $\begin{array}{c}\text { Original system } \\
\mathrm{K}_{\mathrm{p}_{-} \mathrm{PLL}}=30, \mathrm{~K}_{\mathrm{i}_{\mathrm{B} P L L}}=500 \\
\mathrm{~K}_{\mathrm{Pccsc}}=0.5 \text { and } \mathrm{K}_{\text {Iccsc }}=50\end{array}$ & $\begin{array}{l}\text { Increased PLL gains } \\
\qquad \begin{array}{c}\left(\mathrm{K}_{\mathrm{p} \_ \text {PLL }}=300,\right. \\
\left.\mathrm{K}_{\mathrm{i} \_ \text {PLL }}=5000\right)\end{array}\end{array}$ & $\begin{array}{c}\text { Increased CCSC } \\
\text { gains } \\
\mathrm{K}_{\text {Pccsc }}=10, \mathrm{~K}_{\text {Iccsc }}=50\end{array}$ \\
\hline$-14.56 \pm \mathrm{j} 313.2$ & $-6.98 \pm j 317.0$ & $-13.63 \pm j 314.7$ \\
\hline$-17.82 \pm \mathrm{j} 129.5$ & $-33.74 \pm \mathrm{j} 101.3$ & $-9.48 \pm \mathrm{j} 133.9$ \\
\hline
\end{tabular}
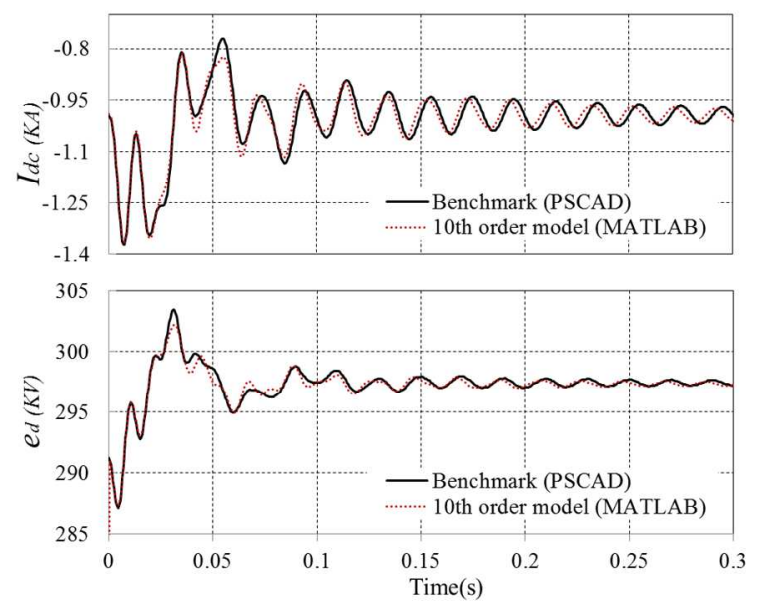

Fig. 10. 10h order model verification with higher PLL gains.

\section{F. Dynamic study of influence of CCSC gains}

The dynamics system with different CCSC gains is also tested, and the linearized model shows very good matching, as seen in Fig. 11. Here, the proportional CCSC gain is increased 20 times which reduces damping of the mode at around $20 \mathrm{~Hz}$, as shown in Table I.

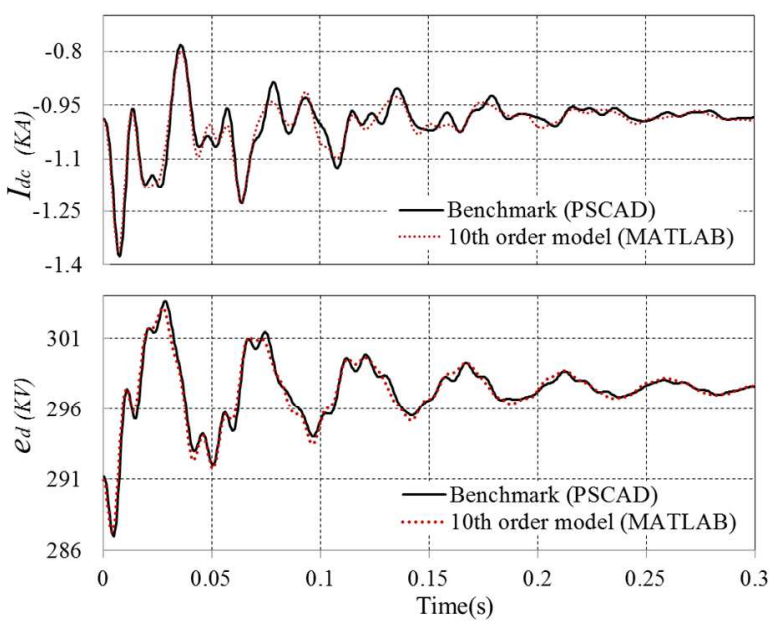

Fig. $11.10^{\text {th }}$ order model verification with higher CCSC gains. 


\section{CONCLUSION}

A $10^{\text {th }}$ order dynamic linearized small-signal model for MMC is proposed. It is concluded that non-linear multiplication terms can be represented directly in DQ frame, and D2Q2 frame rotating at double the fundamental frequency based on the special expressions for DQ frame algebra.

A $6^{\text {th }}$ order dynamic linearized model is proposed as a reduced order model by considering the effect of CCSC on MMC but ignoring the second harmonic modulation indices and circulating current. A further reduced $2^{\text {nd }}$ order model is also proposed by introducing an equivalent series capacitance, $C_{M M C}$, on the converter $\mathrm{AC}$ side.

The accuracy of the 3 models is verified against a detailed benchmark model in PSCAD in both: time and frequency domains. The tests show excellent accuracy for the $10^{\text {th }}$ order and a reduced accuracy for the $6^{\text {th }}$ order model. The $2^{\text {nd }}$ order model shows lowest accuracy but it has advantage in simplicity.

\section{APPENDIX I. DQ FRAME SIGNAL MULTIPLICATION}

Starting with time domain expression for two generic signals $X(t)$ and $Y(t)$, each consisting of zero sequence, fundamental component and second harmonic:

$$
\begin{aligned}
& X(t)=X_{0}+X_{D} \cos \omega t+X_{Q} \sin \omega t+X_{D 2} \cos 2 \omega t+X_{Q 2} \sin 2 \omega t \\
& Y(t)=Y_{0}+Y_{D} \cos \omega t+Y_{Q} \sin \omega t+Y_{D 2} \cos 2 \omega t+Y_{Q 2} \sin 2 \omega t
\end{aligned}
$$

By expanding the $\mathrm{ABC}$ frame expression for product $Z(t)=X(t) \times Y(t)$ and analyzing each term (neglecting $3^{\text {rd }}$ and $4^{\text {th }}$ harmonics) the following is obtained:

$$
\begin{aligned}
& Z(t)=\underbrace{\left(X_{0} Y_{0}+\frac{X_{\mathrm{D}} Y_{\mathrm{D}}}{2}+\frac{X_{\mathrm{Q}} Y_{\mathrm{Q}}}{2}+\frac{X_{D 2} Y_{D 2}}{2}+\frac{X_{Q 2} Y_{Q 2}}{2}\right)}_{Z_{0}}+ \\
& \underbrace{\left(X_{\mathrm{D}} Y_{0}+X_{0} Y_{D}+\frac{1}{2} X_{D 2} Y_{D}+\frac{1}{2} X_{Q 2} Y_{Q}+\frac{1}{2} Y_{D 2} X_{D}+\frac{1}{2} Y_{Q 2} X_{Q}\right)}_{Z_{d}} \cos \omega t \\
& +\underbrace{\left(X_{\mathrm{Q}} Y_{0}+X_{0} Y_{Q}-\frac{1}{2} X_{D 2} Y_{Q}+\frac{1}{2} X_{Q 2} Y_{D}-\frac{1}{2} Y_{D 2} X_{Q}+\frac{1}{2} Y_{Q 2} X_{D}\right)}_{Z_{q}} \sin \omega t \\
& +\underbrace{\left(\frac{X_{\mathrm{D}} Y_{\mathrm{D}}}{2}-\frac{X_{\mathrm{Q}} Y_{Q}}{2}+X_{D 2} Y_{0}+Y_{D 2} X_{0}\right)}_{Z_{d 2}} \cos (2 \omega t) \\
& +\underbrace{\left(\frac{X_{\mathrm{Q}} Y_{\mathrm{D}}}{2}+\frac{X_{\mathrm{D}} Y_{\mathrm{Q}}}{2}+X_{Q 2} Y_{0}+Y_{Q 2} X_{0}\right)}_{Z_{q 2}} \sin (2 \omega t)
\end{aligned}
$$

\section{REFERENCES}

[1] A. Antonopoulos, L. Angquist, and H. Nee, "On Dynamics and Voltage Control of the Modular Multilevel Converter," in Proc. 2009 Power Electronics and Applications (EPE), pp. 1-10.

[2] M. Hagiwara, and H. Akagi, "Control and Experiment of PulsewidthModulated Modular Multilevel Converters" IEEE Transactions on Power Electronics, vol.24, no.7, pp.1737-1746, 2009.

[3] S. Allebrod, R. Hamerski, and R. Marquardt, "New transformerless, scalable Modular Multilevel Converters for HVDC-transmission," IEEE Power Electronics Specialists Conference, 2008, pp.174-179.

[4] U. N. Gnanarathna, A.M. Gole, and R.P. Jayasinghe, "Efficient Modeling of Modular Multilevel HVDC Converters (MMC) on
Electromagnetic Transient Simulation Programs," IEEE Transactions on Power Delivery, vol.26, no.1, pp.316-324, Jan. 2011.

[5] J. Peralta, H. Saad, S. Dennetiere, J. Mahseredjian, and S. Nguefeu, "Detailed and Averaged Models for a 401-Level MMC-HVDC System," IEEE Transactions on Power Delivery, vol.27, no.3, pp.1501-08, July 2012.

[6] H. Saad, J. Peralta, S. Dennetiere, and J. Mahseredjian, "Dynamic Averaged and Simplified Models for MMC-Based HVDC Transmission Systems", IEEE Transactions on Power Delivery, vol. 28, pp. 17231730, July 2013.

[7] S. R. Deore, P. B. Darji and A. M. Kulkarni, "Dynamic Phasor Modeling of Modular Multi-level Converters", 7th IEEE International Conference on Industrial and Information Systems (ICIIS), Aug. 2012.

[8] D. Jovcic and A. Jamshidifar "Phasor Model of Modular Multilevel Converter with Circulating Current Suppression Control" IEEE Transactions on PD, early access DOI: $10.1109 /$ TPWRD.2014.2372780

[9] D. Jovcic, N. Pahalawaththa, M. Zavahir "Stability Analysis of HVDC Control Loops," IEE Proceedings Generation, Transmission and Distribution, vol. 146, no 2, pp. 143-148, March 1999.

[10] W. Juanjuan, F. Chuang, and Z. Yao, "Design of WAMS-Based Multiple HVDC Damping Control System," IEEE transactions on smart grid, vol. 2, no. 2, pp. 363-374, June 2011.

[11] W. Lin and D. Jovcic, "Average modelling of medium frequency DC/DC converters in dynamic studies" IEEE Transactions on Power Delivery, vol. 30, no. 1, pp 281-289, January 2015.

[12] Q. Tu, Z. Xu, and J. Zhang, "Circulating current suppressing controller in modular multilevel converter", IECON Proceedings (Industrial Electronics Conference), art. no. 5675048, pp. 3198-3202.

[13] D. Jovcic, L.A. Lamont,L. Xu, "VSC Transmission Model for Analytical Studies", IEEE Power Engineering Society General Meeting, 2003, IEEE, Volume: 3, 13-17 July 2003, Pages:1737 - 1742

\section{BIOGRAPHIES}

Ali akbar Jamshidifar obtained his B.Sc., M.Sc., and Ph.D. degrees in Control Engineering from Sharif University of Technology, Iran University of Science and Technology, and Amirkabir University of Technology in 1992, 1996, and 2008 respectively. He has been as a researcher with Iranian Research Organization for Science and Technology (IROST) since 1999. He is currently a research fellow with the University of Aberdeen, Scotland. His research interests include the modeling and control of HVDC.

Dragan Jovcic (SM'06, M'00, S'97) obtained a Diploma Engineer degree in Control Engineering from the University of Belgrade, Yugoslavia in 1993 and a Ph.D. degree in Electrical Engineering from the University of Auckland, New Zealand in 1999. He is currently a professor with the University of Aberdeen, Scotland where he has been since 2004. In 2008 he held visiting professor post at McGill University, Montreal, Canada. He also worked as a lecturer with University of Ulster, in the period 2000-2004 and as a design Engineer in the New Zealand power industry in the period 1999-2000. His research interests lie in the FACTS, HVDC, DC grids and control systems. 